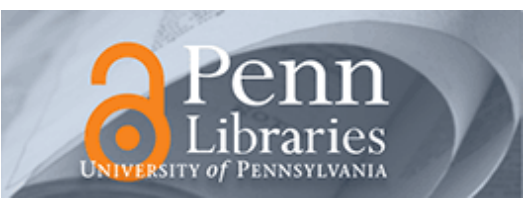

University of Pennsylvania ScholarlyCommons

December 2004

\title{
Correlation of properties with preferred orientation in coagulated and stretch-aligned single-wall carbon nanotubes
}

Stéphane Badaire

Centre de Recherche Paul Pascal-CNRS

Vincent Pichot

Laboratoire de Physique des Solides (UMR CNRS 8502)

Cécile Zakri

Centre de Recherche Paul Pascal-CNRS

Philippe Poulin

Centre de Recherche Paul Pascal-CNRS

Pascale Launois

Laboratoire de Physique des Solides (UMR CNRS 8502)

See next page for additional authors

Follow this and additional works at: https://repository.upenn.edu/mse_papers

\section{Recommended Citation}

Badaire, S., Pichot, V., Zakri, C., Poulin, P., Launois, P., Vavro, J., Guthy, C., Chen, M., \& Fischer, J. E. (2004). Correlation of properties with preferred orientation in coagulated and stretch-aligned single-wall carbon nanotubes. Retrieved from https://repository.upenn.edu/mse_papers/52

Postprint version. Published in Journal of Applied Physics, Volume 96, Issue 12, December 15, 2004, pages 7509-7513.

Publisher URL: http://dx.doi.org/10.1063/1.1810640

This paper is posted at ScholarlyCommons. https://repository.upenn.edu/mse_papers/52

For more information, please contact repository@pobox.upenn.edu. 


\title{
Correlation of properties with preferred orientation in coagulated and stretch- aligned single-wall carbon nanotubes
}

\begin{abstract}
We report structure-property correlations in single wall carbon nanotube (SWNT) fibers, among electrical, thermal and chemical parameters with respect to stretch-induced preferential SWNT alignment along the fiber axis. Purified HiPco tubes are dispersed with the aid of an anionic surfactant and coagulated in the co-flowing stream of an adsorbing polymer. The fibers are then dried, rewetted under tensile load and redried to improve the alignment. Complete removal of the polymer was assured by annealing in hydrogen at $1000^{\circ} \mathrm{C}$. The degree of alignment was determined by $\mathrm{x}$-ray scattering from individual fibers using a 2-dimensional detector. The half width at half maximum (HWHM) describing the axially symmetric distribution of SWNT axes decreases linearly from $27.5^{\circ}$ in the initial extruded fiber to $14.5^{\circ}$ after stretching by $80 \%$. The electrical resistivity $\rho$ at $300 \mathrm{~K}$ decreases overall by a factor $\sim 4$ with stretching, for both as-spun composite and polymer-free annealed fibers. However, the temperature dependence $\rho(T)$ is markedly different for the two, implying different electron transport mechanisms with and without the polymer. Thermal conductivity also improves with increasing alignment, while the absolute values are limited by the disordered network of finite length tubes and bundles. Comparisons are made with results from similar fibers spun from oleum, and with magnetically aligned buckypapers.
\end{abstract}

\section{Comments}

Postprint version. Published in Journal of Applied Physics, Volume 96, Issue 12, December 15, 2004 , pages 7509-7513.

Publisher URL: http://dx.doi.org/10.1063/1.1810640

\section{Author(s)}

Stéphane Badaire, Vincent Pichot, Cécile Zakri, Philippe Poulin, Pascale Launois, Juraj Vavro, Csaba Guthy, Michelle Chen, and John E. Fischer 


\title{
CORRELATION OF PROPERTIES WITH PREFERRED ORIENTATION IN COAGULATED AND STRETCH- ALIGNED SINGLE WALL CARBON NANOTUBES
}

\author{
Stéphane Badaire ${ }^{1}$, Vincent Pichot ${ }^{2}$, Cécile Zakri ${ }^{1}$, Philippe Poulin ${ }^{1}$, Pascale Launois ${ }^{2}$, \\ Juraj Vavro ${ }^{3}$, Csaba Guthy ${ }^{3}$, Michelle Chen $^{3}$ and John E. Fischer ${ }^{3}$ \\ ${ }^{1}$ Centre de Recherche Paul Pascal-CNRS \\ Av. Schweitzer, 33600 Pessac France \\ ${ }^{2}$ Laboratoire de Physique des Solides (UMR CNRS 8502), Bât. 510 \\ Université de Paris Sud, 91405 Orsay France \\ 3 Department of Materials Science and Engineering \\ University of Pennsylvania, Philadelphia PA 19104-6272 USA
}

Corresponding authors e-mail address:

fischer@seas.upenn.edu,poulin@crpp-bordeaux.cnrs.fr

\begin{abstract}
We report structure-property correlations in single wall carbon nanotube (SWNT) fibers, among electrical, thermal and chemical parameters with respect to stretch-induced preferential SWNT alignment along the fiber axis. Purified HiPco tubes are dispersed with the aid of an anionic surfactant and coagulated in the co-flowing stream of an adsorbing polymer. The fibers are then dried, rewetted under tensile load and redried to improve the alignment. Complete removal of the polymer was assured by annealing in hydrogen at $1000^{\circ} \mathrm{C}$. The degree of alignment was determined by $\mathrm{x}$-ray scattering from individual fibers using a 2-dimensional detector. The half width at half maximum (HWHM) describing the axially symmetric distribution of SWNT axes decreases linearly from $27.5^{\circ}$ in the initial extruded fiber to $14.5^{\circ}$ after stretching by $80 \%$. The electrical resistivity $\rho$ at $300 \mathrm{~K}$ decreases overall by a factor $\sim 4$ with stretching, for both as-spun composite and polymer-free annealed fibers. However, the temperature dependence $\rho(T)$ is markedly different for the two, implying different electron transport mechanisms with and without the polymer. Thermal conductivity also improves with increasing alignment, while the absolute values are limited by the disordered network of finite length tubes and bundles. Comparisons are made with results from similar fibers spun from oleum, and with magnetically aligned buckypapers.
\end{abstract}

PACS: 81.07.-b, 81.07.De, 71.23.-k, 61.43.-j,65.80.+n 


\section{Introduction}

Macroscopic oriented assemblies of single wall carbon nanotubes (SWNT) are far more attractive for practical applications than the random tangles of bundled tubes typically found in as-grown or purified bulk samples [1]. Fiber forms could be the starting point for the construction of useful structures which retain to some degree the excellent axial properties expected from perfect infinitely long tubes. Nanotube fibers can be spun by injection of a SWNT suspension through an orifice into a co-flowing stream of a coagulating solution $[2,3]$. This general principle can be simply achieved by mixing an aqueous surfactant-stabilized SWNT dispersion into an aqueous solution that contains a polymer such as polyvinyl alcohol (PVA) as coagulating agent. The resultant fibers offer promise for high strength, light weight, thermally and electrically conducting structural elements at lower cost than other nanotube forms. Industrial-scale continuous processing appears feasible. Mechanical [4,5], electrical and thermal [6] properties will depend on the degree of SWNT alignment induced by fiber synthesis and post-processing. Similar fibers can also be spun from strong acid suspensions [7].

In this paper we study the preferred orientation of PVA/SWNT fibers which are stretch-aligned after spinning $[5,8]$, and correlate their structural features with electrical resistivity and thermal conductivity. The degree of nanotube orientation with respect to the fiber axis is characterized by $\mathrm{X}$ ray scattering $[5,6,8,9]$. The angular distribution of the scattered intensity can be modeled by a Gaussian function, the half width at half maximum (HWHM) of which decreases as the postspinning drawing ratio increases. Quantitatively, HWHM varies from $27.5^{\circ}$ for un-stretched fibers to $14.5^{\circ}$ for fibers stretched by $\delta \mathrm{L} / \mathrm{L} \sim 80 \%$. Resistivity at room temperature for such fibers is shown to decrease by more than a factor 3 as the nanotube alignment is improved. We also show that annealing the fibers at high temperature, to remove the insulating PVA, allows the resistivity to be significantly reduced, by almost four orders of magnitude. Resistivity measurements from 1.4 to $300 \mathrm{~K}$ reveal more information on these structurally disordered conductors. Neat fibers contain up to $50 \mathrm{wt} \%$ PVA and thus can be considered composites of conducting fillers in an insulating matrix. The resistivity is thus very high and strongly temperature-dependent. We find $\rho(4 \mathrm{~K}) / \rho(297 \mathrm{~K}) \sim 10^{6}$. The temperature dependence $\rho(\mathrm{T})$ fits very well to Mott-type phonon-assisted variable range hopping (VRH) but with unphysical parameters. For stretch-aligned fibers after annealing, excellent fits to 3D weak localization (3DWL) and Coulomb gap variable range hopping (CG-VRH) above and below 25K respectively are obtained. Both are consequences of disorder. The low-T CD-VRH regime indicates the opening of a small gap $\Delta_{\mathrm{c}}$ in the density of states when $\Delta_{\mathrm{c}}>\mathrm{kT}$, signifying a metal-insulator transition [10]. In contrast, the thermal conductivity is insensitive to the presence of PVA, the T dependence being dominated by the heat capacity as is generally observed [11]. The present results show the critical importance of understanding the correlation between the chemical and structural nature of nanotube fibers and their electrical properties.

\section{Experimental}

The details of the principle of the spinning method has been described elsewhere [1-2]. The present fibers are spun from purified HiPco tubes purchased from Carbon Nanotechnologies Inc. 
(batch CM26). The tubes were purified by a single pass of thermal oxidation followed by stirring in warm $\mathrm{HCl}$, which typically reduces residual $\mathrm{Fe}$ catalyst from 6-10 to $<1$ at.\% [12]. Dispersions consisting of $0.3 \mathrm{wt} \%$ carbon nanotubes and $1.2 \mathrm{wt} \%$ sodium dodecyl sulfate (SDS) ionic surfactant were prepared. The dispersion is sonicated to achieve a good homogenization. The coagulating medium is an aqueous solution that contains $5 \mathrm{wt} \%$ PVA. The nanotube dispersion is injected at $50 \mathrm{ml} / \mathrm{h}$ through a conical tip into the polymer solution contained in a rotating bath $(100 \mathrm{rpm})$. The obtained fibers are then washed 3 times in pure water, dried in air at room temperature, and finally stretched, as follows $[5,8]$.

Dried fibers are re-swollen in a 50/50wt mixture of water and acetone and then loaded with a given weight and dried under tensile load. The corresponding elongation was determined for each given weight. This method allows the nanotube alignment within the fibers to be improved $[8,9]$. Finally, a portion of each fiber stretched at different draw ratios is annealed at $1000^{\circ} \mathrm{C}$ in hydrogen to remove the organic polymer. Annealed and non-annealed fibers have been investigated.

Electrical properties were measured and correlated to the fiber texture, which in turn was determined by analysis of synchrotron x-ray fiber diagrams $[1,5,8]$. Resistivity was measured by a four-probe DC technique. Voltage probes were about 2-3 $\mathrm{mm}$ apart. Samples were immersed in $\mathrm{He}$, which could be pumped to $\sim 1.5 \mathrm{~K}$. Thermal conductivity $\kappa$ was measured in the range $10-$ $300 \mathrm{~K}$ using a comparator method [6,11]. In brief, a known heat flow $\mathrm{Q}$ passes through a constantan rod, then the sample, and finally a second constantan rod, to a heat sink. Three differential type $\mathrm{E}$ thermocouples using 0.00025 inch diameter wires are employed to measure temperature drops across the sample and the constantan standards, with small sapphire chips to electrically isolate the differential thermocouple from the electrically conducting fiber. Thermal conductance is obtained by averaging the two ratios of temperature drops across the sample and either constantan, then scaled by a dimensional factor. Averaging the two ratios accounts approximately for radiation losses since one of the two constantans is hotter or colder than the sample. The accuracy of both $\rho$ and $\kappa$ data, estimated as $\pm 30 \%$, is limited by errors in sample dimensions. Sample densities were not determined so no corrections for voids and porosity were made.

\section{Results and Discussion}

The X-ray scattering experiments where performed on single fibers, using synchrotron radiation at LURE, France (beam line D43). To avoid background due to the fluorescence from iron catalyst remaining even after purification, experiments were performed at an energy below the $\mathrm{K}$ absorption edge of iron, corresponding to a wavelength of $1.795 \AA$. We study the azimuthal intensity distribution at wave vectors corresponding to the $(1,0)$ peak of the nanotube bundles as detailed in $[8,9]$. The scattering maxima are perpendicular to the fiber axis, which shows that the nanotube bundles are preferentially aligned along this axis. The half width at half maximum (HWHM), deduced from a Gaussian fit, characterizes the distribution of nanotube bundle orientations in the fiber (together with their possible curvatures).

As shown in Figure 1 and as expected, stretching neat, or as-extruded PVA/SWNT fibers decreases the width of Gaussian fits to x-ray fiber diagrams, essentially monotonically with 
increasing elongation. The neat fiber $(0 \%$ stretch $)$ is already partially aligned due to the elongational shear flow accompanying the spinning process. The degree of alignment, as measured by the HWHM (half width at half-maximum) is slightly better than the best fibers spun from oleum [7] but not as good as is found in dilute SWNT/polymer fibers [13]. In the present experiments, the HWHM of un-stretched fibers is about $27.5^{\circ}$, while at the limiting draw ratio $80^{\circ}$ we find HWHM to be decreased by about a factor of 2 overall, to $14.5^{\circ}$.

Resistivity at room temperature $\rho(297 \mathrm{~K})$ vs. stretch ratio is plotted in Figure 2 for unannealed fibers. A trend is evident: a decrease is found as the fibers are stretched, indicating that improvement of nanotube alignment yields better conductivity. In detail, $\rho$ decreases rapidly at first, then saturates at $\sim 35 \%$ stretch. Surprisingly, this behavior is not reflected in the alignment HWHM vs. stretch\% (Figure 1), suggesting that above an intermediate degree of alignment $\rho(297 \mathrm{~K})$ is limited by some other factor which does not improve with further stretching. For this set of experiments the cross-sectional areas were determined fairly accurately from image analysis of SEM data, so we can safely conclude that improving the HWHM from $27.5^{\circ}$ to $14.5^{\circ}$ reduces $\rho(297 \mathrm{~K})$ by about a factor four.

Selected fibers were annealed in $\mathrm{H}_{2}$ at $1000^{\circ} \mathrm{C}$ after stretching, to remove the insulating PVA and thereby obtain more highly conducting fibers. We showed previously that PVA is indeed entirely removed by such a procedure, while the nanotube alignment is unchanged [9]. In Figure 3 we plot the $297 \mathrm{~K}$ resistivity vs. $\%$ stretch after annealing. The data are more scattered than in Figure 2 because the cross-sections are less accurately known. Despite the scatter, it is unambiguous that removal of the PVA leads to a reduction in $\rho(297 \mathrm{~K})$ by almost four orders of magnitude. This strong variation suggests that the conductivity in neat fibers is limited by the presence of PVA, possibly by reducing the density of contacts between nanotube bundles and/or creating strong tunneling barriers between conducting regions.

To gain more insight into the conduction mechanisms, we performed $\rho(\mathrm{T})$ measurements on a composite fiber stretched $53 \%$ and an annealed one stretched $76 \%$. The data are shown in Figure 4 ; note $\log -\log$ scale. At $300 \mathrm{~K} \rho$ for the composite fiber exceeds that of the annealed one by $4-5$ decades, as noted above. At low temperature the difference is more dramatic. The composite fiber resistance became too high to measure at the lowest temperature. Extrapolating to $2 \mathrm{~K}$ we deduce a ratio of at least 11 decades, strongly suggesting totally different conduction mechanisms in the two morphologies.

It is obvious from the data that disorder dominates charge transport in both states. Furthermore, the disorder is most likely to encompass all 3 dimensions since the fibers consist of coupled and intertwined objects. In particular, we expect that the disorder is not limited to 1-D carrier localization on individual tubes or bundles, nor to defect scattering/trapping on single tubes. The huge effect of PVA, which permeates the inter-bundle volumes in the composite fibers, cannot possibly be 1-D.

First we discuss model fits to $\rho(T)$ of annealed fibers. Figure 5 shows the data and fit to a $\log (\rho)$ vs. $1 / \sqrt{ } \mathrm{T}$ law in the range $1.4-300 \mathrm{~K}$, characteristic of strong localization. The fit is perfect below $25 \mathrm{~K}$ but deviates significantly at higher $\mathrm{T}$. It is tempting to assign this behavior to phonon-assisted (Mott) variable range hopping (VRH) between electron states localized in one 
dimension. As noted above, we believe this is inconsistent with the morphology of these materials. A second phenomenon with the same functional dependence is Coulomb gap VRH, wherein strong electron-electron correlations open a gap at the Fermi energy in the otherwise continuous density of states. This model also predicts $\rho(T)=\rho_{0} \exp \left[\left(T_{0} / T\right)^{1 / 2}\right]$, with $1 / T_{0}$ a measure of the spatial extent of the localized wave function; above $\mathrm{T}_{0}$, the thermal energy is sufficient to thermally excite carriers across the Coulomb gap. ( In the case of phonon-assisted (Mott) $\mathrm{VRH}, \mathrm{kT}_{0}$ is the average energy spacing between localized states.) Above $25 \mathrm{~K}$ the data fit well to a model of weak localization in 3-D: $\rho(T)=1 / \sigma(T)_{3 \mathrm{DWL}}+\rho_{0}$ with $\sigma(\mathrm{T})_{3 \mathrm{DWL}}=\sigma_{0}(1+$ $\left.\left(\mathrm{T} / \mathrm{T}_{0}\right)^{\mathrm{p} / 2}\right)$ (not shown). The exponent $\mathrm{p} / 2$ depends on the details of the phase scattering, typical values ranging from 0.8 to $>2$. We note from Figure 5 that CG-VRH accounts for 3 decades of resistivity variation while the $3 \mathrm{DWL}$ regime covers less than a decade of $\rho(\mathrm{T})$.

Identifying the transport mechanism in composite fibers is less evident. Figure 6 shows the data for an $80 \%$ stretched composite fiber, along with an excellent fit to the generic strong localization formula $\rho \sim \exp (\mathrm{A} / \mathrm{T})^{\mathrm{s}}$ covering $\sim 5$ decades in resistivity. Unfortunately neither $\mathrm{A}$ nor s make physical sense, for either Mott or CG strong localization. Fitted exponents s are 0.20 \pm 0.03 for 5 samples with different stretch ratios, whereas for CG $\mathrm{s}=0.5$ and for Mott-type VRH $s=1 / 4,1 / 3$ or $1 / 2$ if the dimensionality is 3,2 or 1 respectively. One might argue that the fitted $s$ is sufficiently close to the 3 -D value of $1 / 4$ to be physically acceptable. Unfortunately, the corresponding $\mathrm{kT}_{0}$ values would imply average localized state energy separations ranging from 260 to $2200 \mathrm{eV}$ for different stretch ratios, clearly nonsensical. As an alternative, we offer an interesting and testable speculation: at $\sim 50 \mathrm{wt} \%$ insulating PVA, the composite system is barely above a percolation threshold at high T, and differential thermal expansion of PVA and SWNT conspire to bring the system below percolation at low $\mathrm{T}$, for example by reducing the number of intertube contacts. One test would be to study the effect of variable PVA content by controlling the coagulation bath composition and/or annealing at low temperatures.

Thermal conductivity $\mathrm{\kappa}$ of annealed fiber was measured from 10 to $300 \mathrm{~K}$ in a closed-cycle cryostat. The comparator method requires that the thermal conductance $G$ of the sample be comparable in magnitude to that of the constantan standards. Sufficient fiber length to achieve this criterion was only available for two stretch ratios, namely $21 \%$ and $58 \%$, for which 20 and $24 \sim 2 \mathrm{~mm}$ lengths respectively were assembled in parallel to achieve sufficient $\mathrm{G}$ (sample). The results are shown in Figure 7. $\kappa$ is dominated by phonons [11] so it increases smoothly with temperature as more vibrational modes become occupied. We did not attempt to measure the composite fibers.

Room temperature values are approximately 4 and $10 \mathrm{~W} / \mathrm{mK}$ for low and high stretch ratios respectively, indicating that $\kappa$ is improved by SWNT alignment, in common with electrical conductivity. Absolute values are about half those measured for similar fibers spun from oleum [6], which is surprising since both fiber series involve HiPco SWNT and similar acid purification schemes. A trivial explanation would be different effective sample dimensions due to gross voids and/or microporosity, since in both cases the raw data were not corrected for density. A second possibility is that inter-particle contacts are more favorable in the oleum-based fibers [14]. In any event, all the fiber $\kappa$ values are very low compared to $\kappa$ of graphite parallel to the layers. Density corrections account for a factor of only 2-5 from our previous experience. Also the tubes/bundles in the fiber are not perfectly aligned. The largest $\kappa$ measured to date, 19 
$\mathrm{W} / \mathrm{mK}$, was obtained from a fiber with mosaic HWHM of $21^{\circ}$ [6], about half the value for the best magnetic field-aligned buckypaper: $48 \mathrm{~W} / \mathrm{m}-\mathrm{K}$ uncorrected for density, HWHM $=16^{\mathrm{o}}$ [15].

The SWNT in buckypaper sample were grown by pulsed laser vaporization, which generally produces longer tubes than the HiPco process. The most important reason for the rather low fiber $\kappa$ 's achieved thus far is probably the aforementioned thermal barriers between bundles etc. which would reduce the mean free path for phonon scattering.

\section{Conclusions}

We have studied in this work the electrical and thermal properties of carbon nanotube fibers formed by coagulation spinning. Clear correlations between structure and properties are evidenced. Both electrical and thermal conductivity of neat or composite fibers can be improved upon alignment of the carbon nanotubes. The relative improvement is rather modest, by a factor 3 to 4 for electrical conductivity, for fibers having the same chemical nature. In sharp contrast, annealing the fibers to remove insulating polymers and obtain neat nanotube fibers, leads to significant increase of the conductivity, by several orders of magnitude, and to differences in the intrinsic conductivity mechanisms. While some aspects remain to be clarified, this work demonstrates the critical importance of the structure and chemical composition of nanotube fibers for their transport properties. Nanotubes fibers are potentially useful for the future development of conducting textiles, composites and electrochemical sensor or actuators. We hope that the present results will help in optimizing the conducting properties of nanotubes fibers for these and other applications.

\section{Acknowledgements}

Research at Penn was supported by the US Department of Energy Grant No. DE-FG0298ER45701 and the Office of Naval Research (DURINT) Grant No. N00014-01-1-0789. We thank D. Durand for her help during the synchrotron measurements, M. Maugey and A. Derré for their contribution in the formulation and thermal treatments of the fibers.

\section{References}

1. P. Launois and P. Poulin, Encyclopedia of Nanoscience and Nanotechnology 4, 1 (2004)

2. B. Vigolo, A. Pénicaud, C. Coulon, C. Sauder, R. Pailler, C. Journet, P. Bernier and P. Poulin., Science 290, 1331 (2000).

3. P. Poulin, B. Vigolo and P. Launois, Carbon 40, 1741 (2002).

4. A. B. Dalton, S. Collins, E. Muňoz, J.M. Razal, V.H. Ebron, J.P. Ferraris, J.N. Coleman, B. G. Kim and R.H. Baughman, Nature 423, 703 (2003).

5. B. Vigolo, M. Lucas, P. Launois, P. Bernier and P. Poulin, Applied Physics Letters 81, 1210 (2002).

6. W. Zhou, J. Vavro, C. Guthy, K.I. Winey, J.E. Fischer, L.M. Ericson, S. Ramesh, R. Saini, V.A. Davis, C. Kittrell, M. Pasquali, R.H. Hauge and R.E. Smalley, J. Appl. Phys. 95, 649 (2004).

7. L. M. Ericson, H. Fan, H. Peng, V. A. Davis, W. Zhou, J. Sulpizio, Y. Wang, R. Booker, J. Vavro, C. Guthy, A. N. G. Parra-Vasquez, M. J. Kim, S.Ramesh, R. K. Saini, C. Kittrell, G. Lavin, H. Schmidt, W. W. Adams, W. E. Billups, M. Pasquali, W.-F. Hwang, R. Hauge, J. E. Fischer and R. E. Smalley, Science 305, 1447 (2004). 
8. M. Lucas, B. Vigolo, S. Badaire, D. Le Bolloc'h, A. Marucci, D. Durand, M. Hamilton, C. Zakri, P. Poulin and P. Launois, AIP Conf. Proc. 633, H. Kuzmany, J. Fink, M. Mehring and S. Roth Eds., 579 (2002).

9. P. Launois, A. Marucci, B. Vigolo, P. Bernier, A. Derré, P. Poulin, J. Nanosci. Nanotech. 1, $125(2001)$.

10. J. Vavro, J. Kikkawa and J. E. Fischer, Phys. Rev. B (BT8910 in press).

11. J. Hone, M. C. Llaguno, M. Biercuk, A. T. Johnson, B. Batlogg, Z. Benes and J. E. Fischer, Applied Physics A 74, 339 (2002).

12. W. Zhou, Y. H. Ooi, R. Russo, P. Papanek, D. E. Luzzi, J. E. Fischer, M. J. Bronikowski, P.A. Willis and R. E. Smalley, Chem. Phys. Letters 350, 6-10 (2001).

13. R. Haggenmueller, W. Zhou, J.E. Fischer and K.I. Winey, J. Nanosci. Nanotech. 3, 105 (2003)

14. Davis, V. A.; Ericson, L. M.; Parra-Vasquez, A. N. G.; Fan, H.; Wang, Y.; Prieto, V.;

Longoria, J. A.; Ramesh, S.; Saini, R. K.; Kittrell, C.; Billups, W. E.; Adams, W. W.; Hauge, R. H.; Smalley, R. E.; Pasquali, M.; Macromolecules 2004; 37(1); 154-160.

15. J. E. Fischer, W. Zhou, J. Vavro, M. C. Llaguno, C. Guthy, R. Haggenmueller, M. J.

Casavant, D. E. Walters and R. E. Smalley, J. Appl. Physics 93, 2157 (2003).

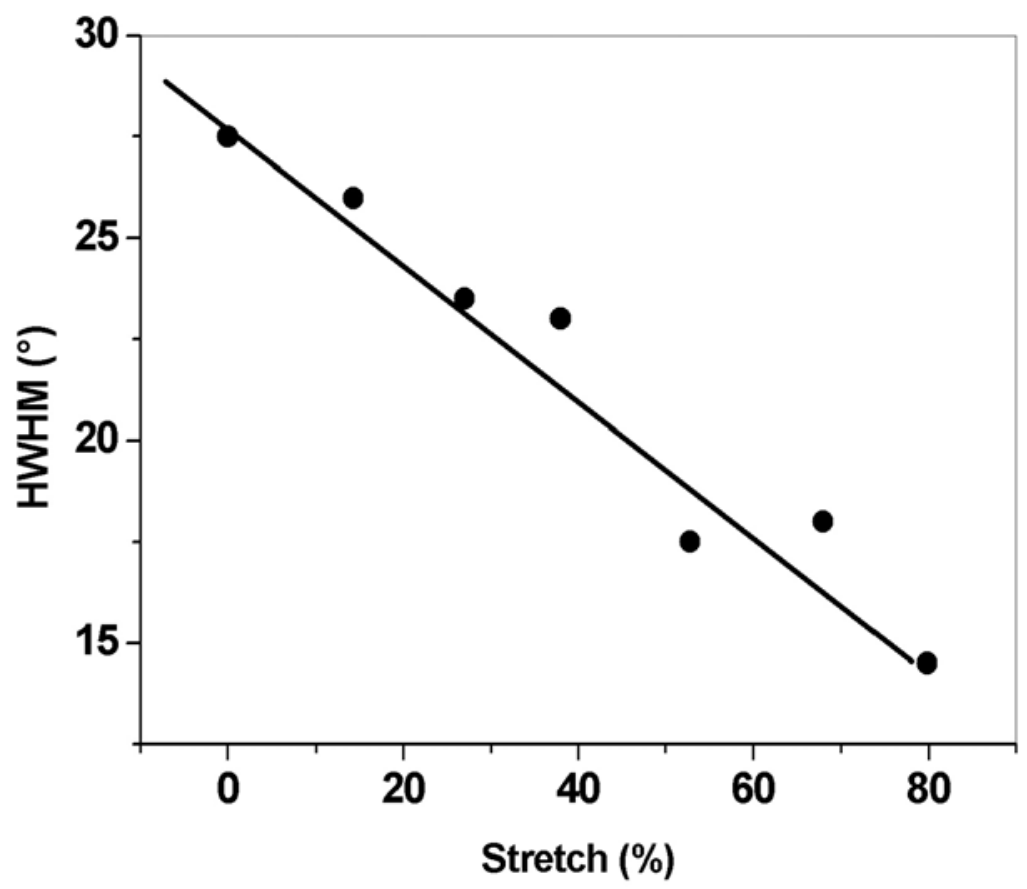

Figure 1: Gaussian half width at half maximum (HWHM) vs. stretch ratio for PVA/SWNT composite nanotube fibers. The initial alignment (stretch $\%=0$ ) results from extrusional flow during spinning. This improves due to extensional flow under tension. 


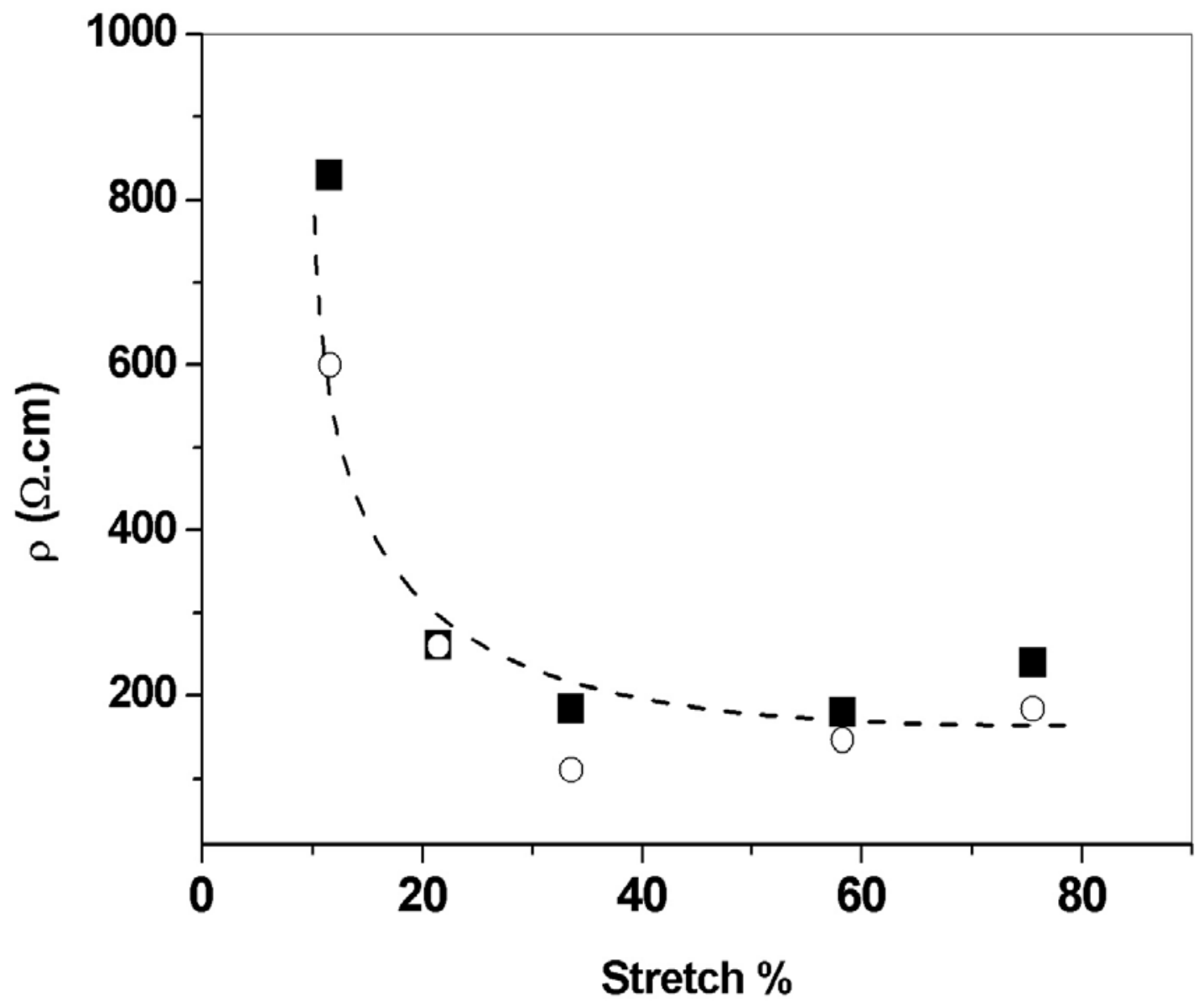

Figure 2: 4-probe electrical resistivity at room temperature vs. stretch ratio for composite nanotube fibers. The experiments have been conducted on two series of samples (black squares, open circles). Accurate cross-sectional areas were obtained from SEM image analysis. The resistivity decreases with stretch ratio by a factor between 3 and 4 for the two set of data. The absolute value is intermediate between all-SWNT fibers and polymer composites. The dashed line is a guide to the eye. 


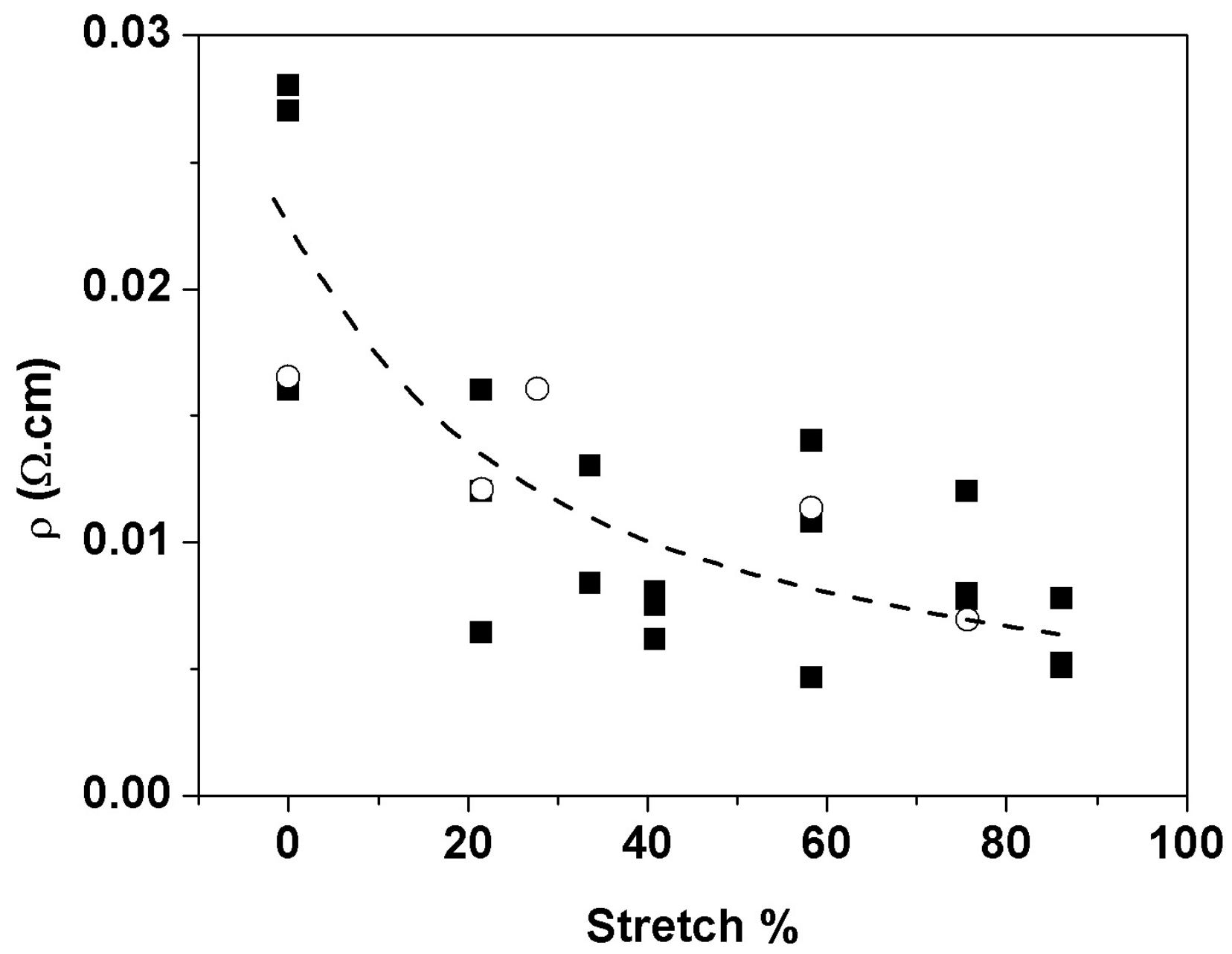

Figure 3: 4-probe electrical resistivity at room temperature vs. stretch ratio for 2 sets of annealed nanotube fibers. Cross-sections were determined from optical microscopy and are therefore less accurate than in Figure 2. The trend of decreasing $\rho$ with increasing stretch $\%$ is less apparent than in Figure 2. The resistivity decreases dramatically upon removal of the nonconducting PVA. 


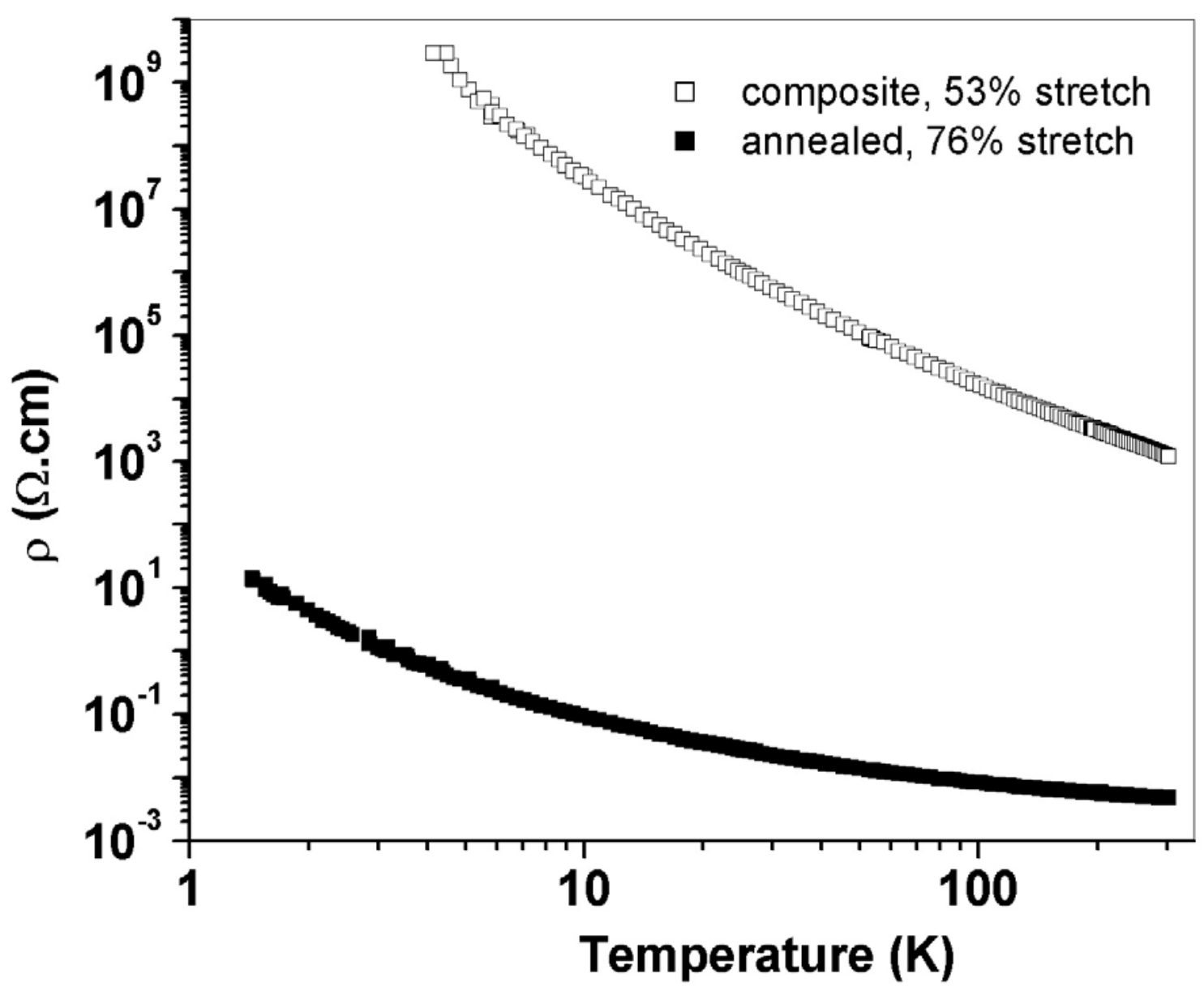

Figure 4: Resistivity vs. temperature for composite (53\% stretch) and annealed (76\% stretch) nanotube fibers (note $\log -\log$ scale). Note that $\rho(\mathrm{T})$ for the composite fiber is more strongly divergent as $\mathrm{T} \rightarrow 0$ as compared to the annealed one. 


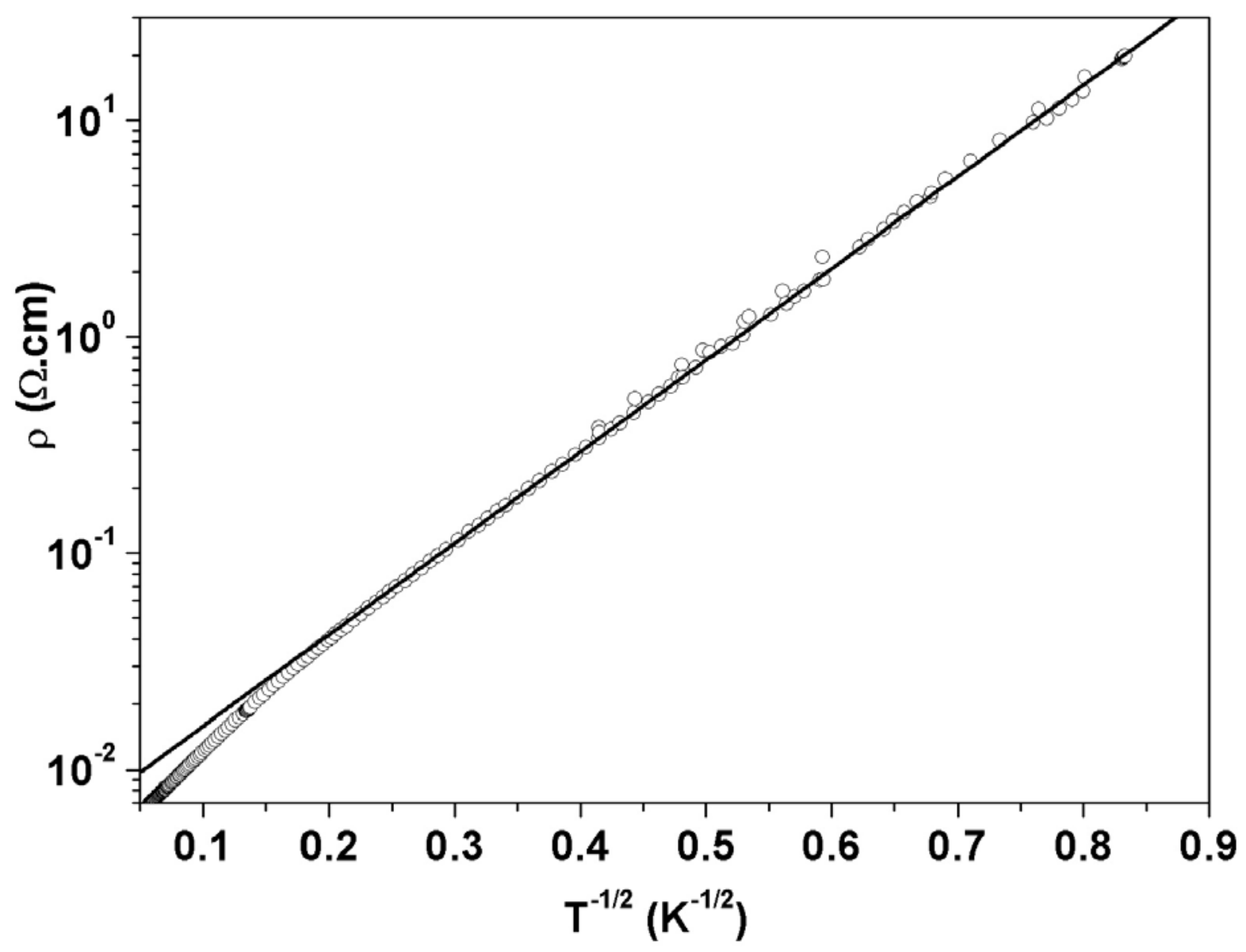

Figure 5: Fit to a model of strong localization (solid curve) to the data from annealed $76 \%$ stretch sample (solid squares). The fit is excellent at low temperatures $\mathrm{T}<25 \mathrm{~K}$ and over 3 decades of $\rho$. The characteristic temperature in the factor $\exp \left(\mathrm{T}_{0} / \mathrm{T}\right)^{1 / 2}$ is $95 \mathrm{~K}$, or $\sim 8 \mathrm{meV}$, which we associate with a Coulomb gap (see text). 


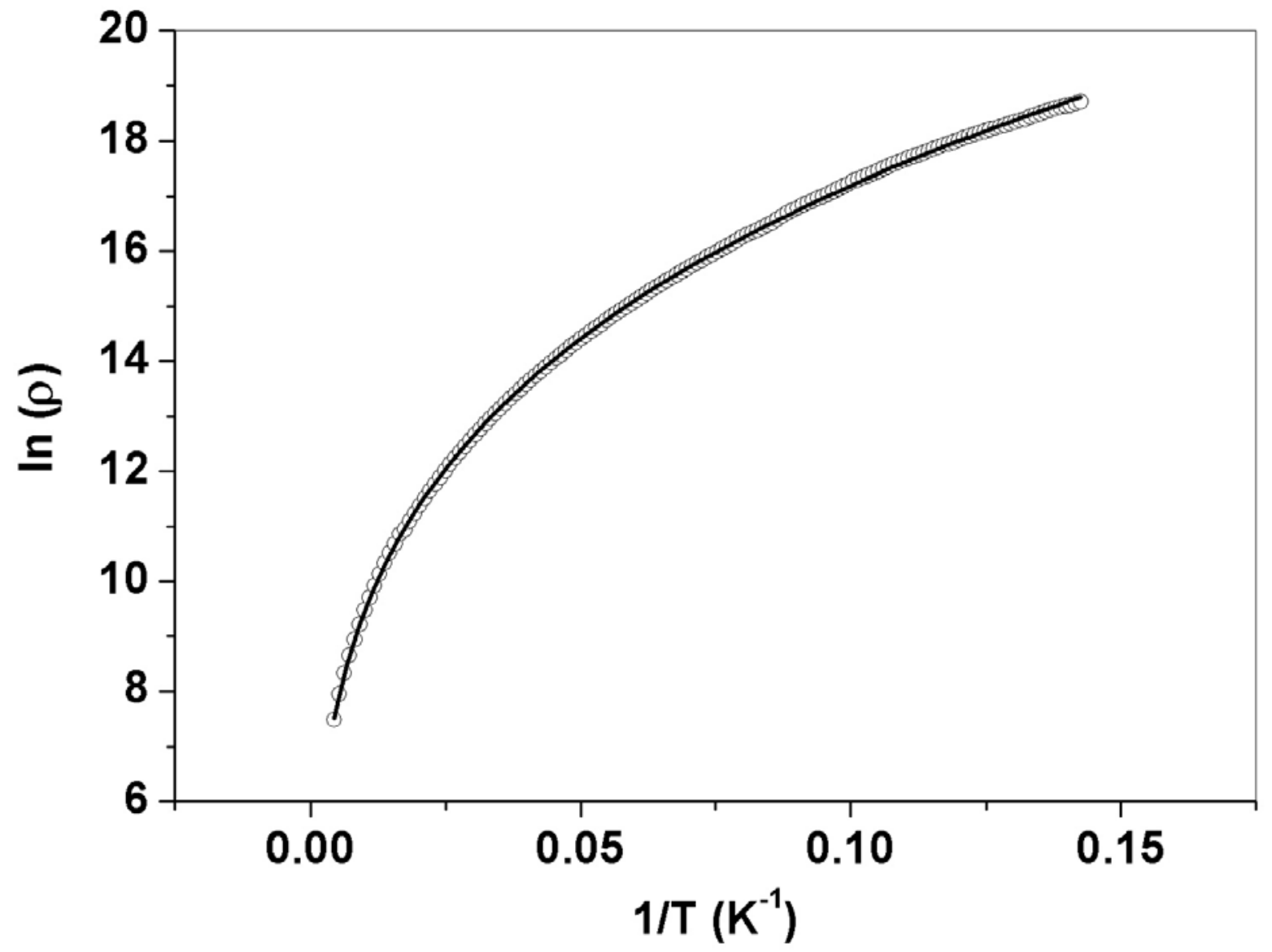

Figure 6: Fit using a generic strong localization model (solid curve) to the data from a PVA/SWNT composite $80 \%$ stretched sample (solid squares). The fit is excellent at all temperatures but the parameters are unphysical for either phonon-assisted or Coulomb gap VRH (see text). 


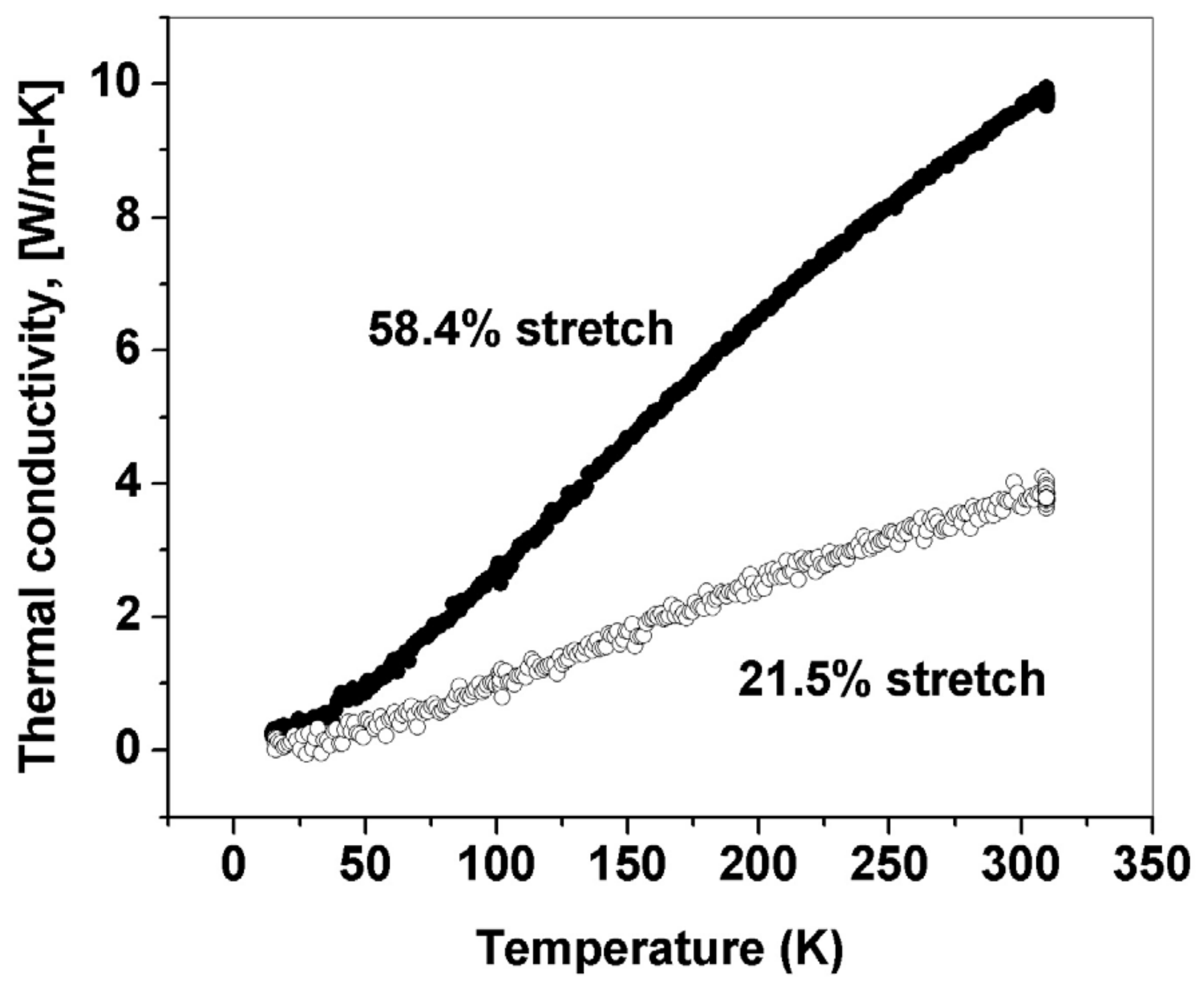

Figure 7: Thermal conductivity vs. temperature for annealed nanotube fibers which have been stretch-aligned to $21 \%$ (lower curve) and 58\% (upper curve). Additional alignment of the latter yields a fator $\sim 2$ further enhancement of $\kappa$. The temperature dependence is dominated by that of the lattice specific heat, as is generally observed. 